\title{
A real-time anatomy identification via tool based on artificial intelligence for ultrasound-guided peripheral nerve block procedures: an accuracy study
}

\author{
Irfan Gungor ${ }^{1} \cdot$ Berrin Gunaydin $^{1}$ [ - Suna O. Oktar ${ }^{2} \cdot$ Beyza M.Buyukgebiz $^{1} \cdot$ Selin Bagcaz ${ }^{1} \cdot$ Miray Gozde Ozdemir $^{1}$. \\ Gozde Inan ${ }^{1}$
}

Received: 14 February 2021 / Accepted: 7 May 2021 / Published online: 19 May 2021

(c) Japanese Society of Anesthesiologists 2021

\begin{abstract}
We aimed to assess the accuracy of an artificial intelligence (AI)-based real-time anatomy identification software specifically developed to ease image interpretation intended for ultrasound-guided peripheral nerve block (UGPNB). Forty healthy participants (20 women, 20 men) were enrolled to perform interscalene, supraclavicular, infraclavicular, and transversus abdominis plane (TAP) blocks under ultrasound guidance using AI software by anesthesiology trainees. During block practice by a trainee, once the software indicates $100 \%$ scan success of each block associated anatomic landmarks, both raw and labeled ultrasound images were saved, assessed, and validated using a 5-point scale by expert validators. When trainees reached $100 \%$ scan success, accuracy scores of the validators were noted. Correlation analysis was used whether the relationship ( $r$ ) according to demographics (gender, age, and body mass index: BMI) and block type exist. The BMI $\left(\mathrm{kg} / \mathrm{m}^{2}\right)$ and age (year) of participants were 22.2 \pm 3 and 32.2 \pm 5.25 , respectively. Assessment scores of validators for all blocks were similar in male and female individuals. Mean assessment scores of validators were not significantly different according to age and BMI except for TAP block, which was inversely correlated with age and BMI $(p=0.01)$. AI technology can successfully interpret anatomical structures in real-time sonography while assisting young anesthesiologists during UGPNB practice.
\end{abstract}

Keywords Regional anesthesia $\cdot$ Peripheral nerve block $\cdot$ ultrasound $\cdot$ Artificial intelligence

Artificial intelligence (AI) has been used in many disciplines including particularly radiology till now [1,2]. Regional anesthesia is a potential area to utilize AI-based solutions to assist practitioners to avoid unwanted nerve, artery, vein, pleural, or peritoneal puncture complications because understanding device operations, image optimization, image interpretation, and visualization of needle insertion and injection of local anesthetic solution is required to overcome experience related barriers and improve nerve block performance [3-7]. To our knowledge, clinical validation of the accuracy of a real-time sonoanatomy identification via AI-supported

Berrin Gunaydin

gunaydin@gazi.edu.tr

1 Department of Anesthesiology and Reanimation, Gazi University Faculty of Medicine, Besevler, 06500 Ankara, Turkey

2 Department of Radiology, Gazi University Faculty of Medicine, Ankara, Turkey ultrasound-guided peripheral nerve block (UGPNB) practice has not been conducted yet. Therefore, we aimed to assess the accuracy of an AI-based real-time anatomy identification specifically developed to ease image interpretation intended for ultrasound-guided interscalene, supraclavicular, infraclavicular, and transversus abdominis plane (TAP) blocks performed by trainees in healthy volunteer subjects.

After we obtained IRB approval with the Pharmaceuticals and Medical Devices Agency of the country's permission (15.12.2020/E.595466), 40 healthy participants $(n=20$ men, $n=20$ women) were informed about AI software validation study. After obtaining written informed consent, participants were recruited and their age and body mass index (BMI) were noted. It was clearly explained that only ultrasound procedures would be practiced without any further block performance. The participants were warned about precautionary pandemic measures against COVID-19. Three anesthesia trainees at the $2 \mathrm{nd}$, 3rd, and 4th years of education scanned ultrasound images. 
An ultrasound device with an 8-13 MHz linear ultrasound probe (General Electric LOGIQ, Wisconsin, USA) was used. AI software (Nerveblox, manufactured by Smart Alfa Teknoloji San. ve Tic. A.Ş., Ankara, Turkey) was installed on an off-the-shelf personal computer (Lenovo V530 Touch screen i5-9400 T 3.4 GHz 8 GB RAM 256 GB SSD) running Linux operating system. An HDMI cable and a video capture adapter were used to feed the ultrasound device's video output into the personal computer in real-time. On the settings section of the software, both "Color Overlay" and "Text Tags" styles were selected under the "Labeling Preferences". The manufacturer claims that when an ultrasound stream is provided, the software can identify the anatomical landmarks in realtime to assist the operator while interpreting the image.

AI software presents a "Scan Success" which is a mathematical representation of the percentage/ratio of the predefined anatomical landmarks labeled on the screen. As per the claim of the manufacturer, when the "Scan Success" reaches $100 \%$, the software-generated labels (color overlays) will confidently represent the anatomical landmarks shown on the ultrasound image. Our study is based on the images where the "Scan Success" gauge shows $100 \%$ are acquired. Thus, when all predefined labeled anatomical landmarks are visualized, scan success of $100 \%$ is recognized. In all subjects, we could reach $100 \%$ Scan Success gauge.

Three trainees practiced/scanned 4 block regions (Fig. 1) on each healthy volunteer one by one and used the freeze function of the ultrasound device when "Scan Success" was $100 \%$. Subsequently, the corresponding raw US image and generated labels were saved by ultrasound and software. A total of 480 raw and labeled image pairs were obtained as a combination of 3 practitioners, 40 healthy volunteers, and 4 block types.
Similar to the study design used in the research conducted by Şendur et al. [8], an experienced anesthesiologist and a radiologist were assigned as a validator (V). For assuring objective decision-making, validators blindly evaluated 480 raw and labeled image pairs. They scored the representation accuracy of each predefined anatomical landmark (Table 1) using a 5-point scale (1: Very Poor, 2: Poor, 3: Good, 4: Very Good, 5: Excellent). Accuracy is defined as expert opinion on how the software-generated landmark labels represent the real anatomy on raw ultrasonography images.

SPSS 25.0 package program was used for analysis. After descriptive statistics, results were expressed as frequency, percentage, or mean \pm standard deviation (sd) where appropriate. Kappa test was conducted to examine the level of agreement between the evaluation scores of two expert validators for selected block types. Independent sample t-test was used to elucidate the accuracy scores when operators reach $100 \%$ scan success. Mean assessment scores of validators in male and female subjects were compared. Correlation analysis was performed whether there was a relationship $(r)$ in the assessment scores of validators between demographics (age and body mass index: BMI) and block type. A P value less than 0.05 was considered as statistically significant.

The mean BMI $\left(\mathrm{kg} / \mathrm{m}^{2}\right)$ and age (year) of the participants were $22.2 \pm 3$ and $32.2 \pm 5.25$, respectively. To prevent complications, predefined anatomical landmarks (including vessels, nerve plexus, and others such as; peritoneumpleura-1st rib) associated with each block type were presented (Table 1). Our results indicated an entire population of images used for the evaluation because all the predefined anatomical landmarks are always available in healthy normal subjects, hence we got evaluable images from all 40 participants.

The mean assessment scores of two validators for TAP block $(\kappa=0.95)$, interscalene block $(\kappa=0.98)$,
Fig. 1 Boundary prediction of intersaclene block (a), supraclavicular block (b), infraclavicular block (c) and TAP block (d) with predefined anatomic landmarks in color-labeled overlay
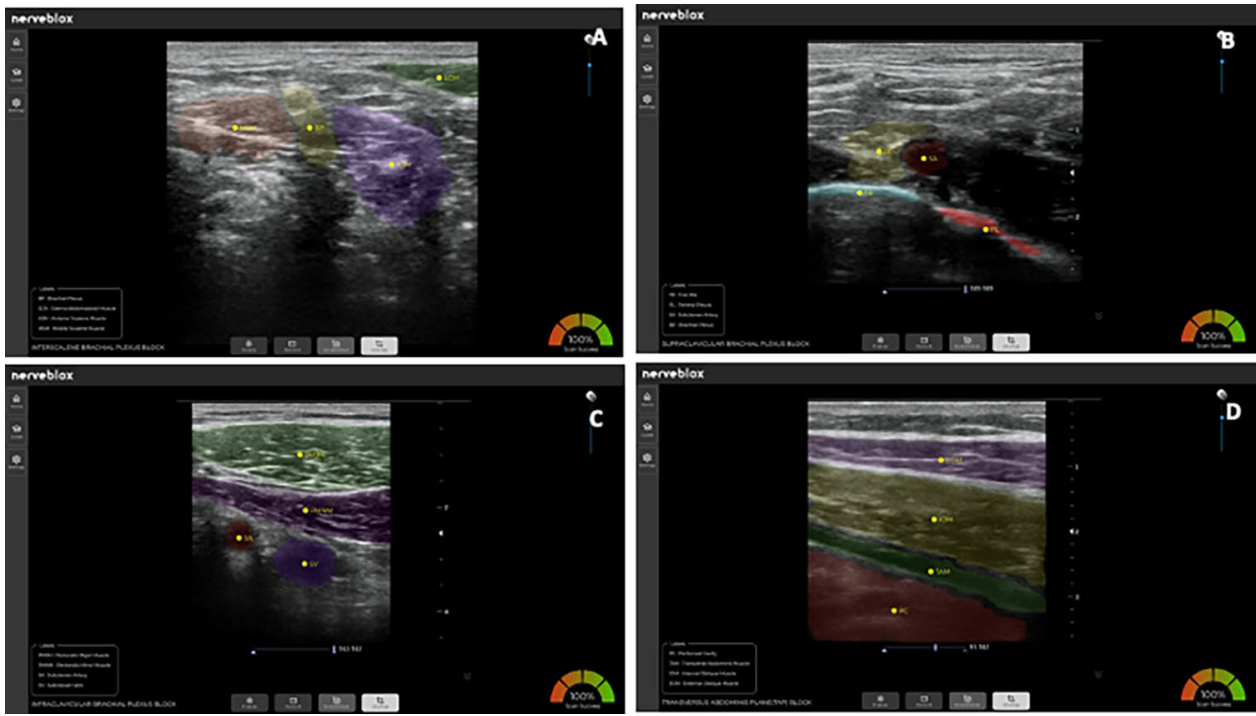
Table 1 Representation of landmark labels of block types and assessment of each validator $(\mathrm{V})$ according to selected block associated anatomical landmarks $($ mean $\pm s d)$

\begin{tabular}{llrrr}
\hline Block type & Predefined anatomical landmarks & V1 & V2 & $p$ \\
\hline Interscalene & Brachial plexus (BP) & $4.84 \pm 0.47$ & $4.92 \pm 0.41$ & 0.96 \\
& Anterior scalene muscle (ASM) & $4.89 \pm 0.31$ & $4.87 \pm 0.33$ & 0.98 \\
& Middle scalene muscle (MSM) & $4.88 \pm 0.37$ & $4.86 \pm 0.35$ & 0.95 \\
& Sternocleidomastoid muscle (SCM) & $4.96 \pm 0.2$ & $4.94 \pm 0.22$ & 0.96 \\
Supraclavicular & First rib (FR) & $5 \pm 0.01$ & $4.98 \pm 0.12$ & 0.96 \\
& Pleura (PL) & $5 \pm 0.01$ & $4.98 \pm 0.12$ & 0.96 \\
& Subclavian artery (SA) & $5 \pm 0.01$ & $4.98 \pm 0.05$ & 0.97 \\
Infraclavicular & Brachial plexus (BP) & $4.9 \pm 0.01$ & $4.98 \pm 0.04$ & 0.99 \\
& Pectoralis major muscle (PMJ) & $5 \pm 0.01$ & $4.97 \pm 0.08$ & 0.95 \\
& Pectoralis minor muscle (PMN) & $5 \pm 0.01$ & $4.98 \pm 0.07$ & 0.96 \\
& Axillary artery (AA) & $5 \pm 0.01$ & $4.99 \pm 0.05$ & 0.99 \\
TAP & Axillary vein (AV) & $4.3 \pm 0.26$ & $4.95 \pm 0.12$ & 0.94 \\
& Transverse abdominis muscle (TAM) & $5 \pm 0.01$ & $4.98 \pm 0.1$ & 0.98 \\
& Internal oblique muscle (IOM) & $4.98 \pm 0.16$ & $4.97 \pm 0.12$ & 0.96 \\
& External oblique muscle (EOM) & $4.98 \pm 0.16$ & $4.96 \pm 0.15$ & 0.92 \\
& Peritoneal cavity (PC) & $4.95 \pm 0.22$ & $4.93 \pm 0.2$ & 0.95 \\
\hline
\end{tabular}

supraclavicular block $(\kappa=0.96)$, and infraclavicular block $(\kappa=0.97)$ associated landmarks were consistent. There was no significant difference in the mean assessment scores of validators between male and female subjects for any block $(p>0.05)$ (Table 1).

The average score of validators for interscalene $(p=0.45$, $r=0.07)$, supraclavicular, and infraclavicular blocks was not significantly correlated by age and BMI $(p>0.05)$. However, assessment of TAP block was inversely correlated with age $(p=0.01, r=-0.38)$ and BMI $(p=0.01, r=-0.26)$. Accuracy of an AI-based software supporting ultrasound-guided selected blocks was achieved in male and female subjects by identifying required anatomic landmarks including nerves, arteries, veins, muscles, pleura, peritoneum, and first rib.

Identifying nerve structures and anatomic landmarks in ultrasound images to prevent complications and interpretation of sonoanatomy are critical steps in UGPNB practice [9-12]. Limited experience in sonoanatomy may lead to nerve injury, local anesthetic toxicity, pneumothorax, and abdominal organ injury complications, though ultrasound guidance provides superior ability to visualize key structures and landmarks. Assistive machine learning systems that focus on architectures and mathematical scores of the AI techniques developed real-time segmentation models for ultrasound-guided femoral block and anatomic identification of lumbar vertebras [13-17]. Our study has been the first to validate an AI software as a medical device rather than a technique/algorithm on selected commonly used peripheral and plane blocks. Hereby, mean accuracy scores for interscalene block (that targets brachial plexus roots; Cervical 5, 6, and 7 by proximal approach) landmarks were 4.84 and 4.90 for 1st and 2nd validators, respectively (Table 1). According to Ding et al. [11], an image segmentation technique was evaluated mathematically and an ultrasound image and neck region was found to be promising and a segmentation algorithm was proposed to identify different tissues on ultrasound images of the interscalene block region.

During supraclavicular block (superior to the subclavian artery and close to pleura) practice, pneumothorax or local anesthetic toxicity might be potential complications of UGPNBs in case of difficult anatomy and accidental artery puncture, respectively [18-21]. To prevent it, color-labeling of pleura was done by AI. The accuracy score for the pleura landmark was assessed as 5.0 and 4.98 by 1 st and 2 nd validators, respectively. The accuracy scores of the subclavian artery and 1st rib landmarks were 5 and 4.98, respectively (Table 1).

During infraclavicular block practice by lateral sagittal approach at brachial plexus level around axillary artery at 3,6 , and 9 o'clock positions, the mean accuracy scores of axillary artery and vein were 5 and 4.99 (for V1 and V2) and 4.93 and 4.95 (for V1 and V2), respectively (Table 1).

In TAP block, local anesthetic solution is injected between muscles [22]. The mean scores of peritoneal cavity landmarks were 4.95/4.93 respectively. Peritoneal puncture and visceral organ injury are potential concerns even under ultrasound guidance.

Arteries and veins (for supraclavicular and infraclavicular blocks), pleura (for supraclavicular block) and peritoneum (for TAP block) images are predefined anatomic landmarks when visible on the screen. Nerveblox software directly visualizes color-labeled structures to distinguish, differentiate and adjust transparency. AI-supported ultrasound use in UGPNB seems to be advantageous to provide training of trainees and enhance experienced instructors' performance. The effect of embedded electronic tutorials on the ultrasound 
machine in terms of speed and accuracy was helpful for novice users to identify anatomic structures [23]. Accuracy of AI software-generated landmark labels in male and female volunteers was demonstrated with a few missing scores (i.e. accuracy score $<5$ ) that could have been due to misaligned borders of some landmarks. However, borders are not known to be precise on the B-mode ultrasound image.

A limitation/weakness of our study might be the lack of volunteers from pediatric/geriatric participants with difficult anatomy of the related block site. Therefore, accuracy for all UGPNBs in different populations needs to be further studied and/or reproduced. Our AI study does not focus on anatomical variations as well as all other block types which the software claims that is capable of. However, we selected the frequently performed blocks in our institution.

In conclusion, the first validation study using UGPNB practice supported by AI-based technology might be a promising assistive tool for interpretation of sonoanatomy to aid real-time immediate decision-making of a particular block associated with predefined anatomic landmarks in healthy volunteers.

\section{References}

1. Lee CS, Tyring AJ, Wu Y, Xiao S, Rokem AS, DeRuyter NP, Zhang Q, Tufail A, Wang RK, Lee AY. Generating retinal flow maps from structural optical coherence tomography with artificial intelligence. Sci Rep. 2019;9(1):5694.

2. Choy G, Khalilzadeh O, Michalski M, Do S, Samir AE, Pianykh OS, Geis JR, Pandharipande PV, Brink JA, Dreyer KJ. Current applications and future impact of machine learning in radiology. Radiology. 2018;288(2):318-28.

3. Sites BD, Chan VW, Neal JM, Weller R, Grau T, KoscielniakNielsen ZJ, Ivani G. The American society of regional anesthesia and pain medicine and the European society of regional anaesthesia and pain therapy joint committee recommendations for education and training in ultrasound-guided regional anesthesia. Reg Anesth Pain Med. 2009;34(1):40-6.

4. Strakowski JA. Ultrasound-guided peripheral nerve procedures. Phys Med Rehabil Clin North Am. 2016;27(3):687-715.

5. Greher M, Retzl G, Niel P, Kamolz L, Marhofer P, Kapral S. Ultrasonographic assessment of topographic anatomy in volunteers suggests a modification of the infraclavicular vertical brachial plexus block. Br J Anaesth. 2002;88(5):632-6.

6. Marhofer P, Greher M, Kapral S. Ultrasound guidance in regional anaesthesia. Br J Anaesth. 2005;94(1):7-17.

7. Kapral S, Krafft P, Eibenberger K, Fitzgerald R, Gosch M, Weinstabl C. Ultrasound-guided supraclavicular approach for regional anesthesia of the brachial plexus. Anesth Analg. 1994;78(3):507-13.

8. Şendur HN, Cindil E, Cerit M, Demir NB, Sendur AB, Oktar SO. Interobserver variability and stiffness measurements of normal common extensor tendon in healthy volunteers using shear wave elastography. Skeletal Radiol. 2019;48(1):137-41.
9. Drukker L, Noble JA, Papageorghiou AT. Introduction to artificial intelligence in ultrasound imaging in obstetrics and gynecology. Ultrasound Obstet Gynecol. 2020;56(4):498-505.

10. Majkowska A, Mittal S, Steiner DF, Reicher JJ, McKinney SM, Duggan GE, Eswaran K, Chen PC, Liu Y, Kalidindi SR, Ding A, Corrado GS, Tse D, Shetty S. Chest radiograph interpretation with deep learning models: assessment with radiologist-adjudicated reference standards and population-adjusted evaluation. Radiology. 2020;294(2):421-31.

11. Ding Y, Yang Q, Wu G, Zhang J, Qin Z (2020) Multiple instance segmentation in brachial plexus ultrasound image using BPMSegNet. arXiv e-prints.

12. Niazi AU, Haldipur N, Prasad AG, Chan VW. Ultrasoundguided regional anesthesia performance in the early learning period: effect of simulation training. Reg Anesth Pain Med. 2012;37(1):51-4.

13. Antonakakis JG, Ting PH, Sites B. Ultrasound-guided regional anesthesia for peripheral nerve blocks: An evidence-based outcome review. Anesthesiol Clin. 2011;29(2):179-91.

14. Bowness J, El-Boghdadly K, Burckett-St LD. Artificial intelligence for image interpretation in ultrasound-guided regional anaesthesia. Anaesthesia. 2020. https://doi.org/10.1111/anae. 15212(online).

15. Smistad E, Iversen DH, Leidig L, Lervik Bakeng JB, Johansen $\mathrm{KF}$, Lindseth F. Automatic segmentation and probe guidance for real-time assistance of ultrasound-guided femoral nerve blocks. Ultrasound Med Biol. 2017;43(1):218-26.

16. Huang C, Zhou Y, Tan W, Qiu Z, Zhou H, Song Y, Zhao Y, Gao $S$. Applying deep learning in recognizing the femoral nerve block region on ultrasound images. Ann Transl Med. 2019;7(18):453. https://doi.org/10.21037/atm.2019.08.61.

17. Hetherington J, Lessoway V, Gunka V, Abolmaesumi P, Rohling R. SLIDE: automatic spine level identification system using a deep convolutional neural network. Int J Comput Assist Radiol Surg. 2017;12(7):1189-98.

18. Jeng CL, Torrillo TM, Rosenblatt MA. Complications of peripheral nerve blocks. Br J Anaesth. 2010;105(Suppl 1):i97-107. https://doi.org/10.1093/bja/aeq273.

19. Orebaugh SL, Kentor ML, Williams BA. Adverse outcomes associated with nerve stimulator-guided and ultrasound-guided peripheral nerve blocks by supervised trainees: update of a single-site database. Reg Anesth Pain Med. 2012;37(6):577-82.

20. Barrington MJ, Kluger R. Ultrasound guidance reduces the risk of local anesthetic systemic toxicity following peripheral nerve blockade. Reg Anesth Pain Med. 2013;38(4):289-99.

21. El-Boghdadly K, Pawa A, Chin KJ. Local anesthetic systemic toxicity: current perspectives. Local Reg Anesth. 2018;11:35-44.

22. Sai HC, Yoshida T, Chuang TY, Yang SF, Chang CC, Yao HY, Tai YT, Lin JA, Chen KY. Transversus abdominis plane block: an updated review of anatomy and techniques. Biomed Res Int. 2017;2017:8284363. https://doi.org/10.1155/2017/8284363 (Epub 2017 Oct 31).

23. Wegener JT, Van Doorn CT, Eshuis JH, Hollmann MW, Preckel B, Stevens MF. Value of an electronic tutorial for image interpretation in ultrasound-guided regional anesthesia. Reg Anesth Pain Med. 2013;38(1):44-9.

Publisher's Note Springer Nature remains neutral with regard to jurisdictional claims in published maps and institutional affiliations. 